\title{
Discursos y prácticas de la discriminación positiva para políticas indígenas en educación superior
}

\author{
Discourses and practices of positive discrimination for indigenous policies in higher education \\ Vanessa Jara-Labarthé (vanedk@gmail.com) Escuela de Trabajo Social, Universidad de Tarapacá (Arica, \\ Chile) ORCID: 0000-0003-1230-7395
}

\begin{abstract}
Positive discrimination, also known as affirmative action, has been positioned as a framework from which actions have been developed to reduce inequalities and increase opportunities in the educational field. Universities, at the international level, have not been left out, and have implemented actions to improve access, retention and graduation of indigenous students. This article discusses the history, the rationale and the tensions of this concept, and discusses some international debates about its effectiveness. Finally, it is proposed a reflection on these actions, as policy and discourse that allow us to broaden our critical view of the actions implemented by universities to reduce educational gaps.
\end{abstract}

Key words: universities, educational trajectories, educational policies, indigenous students, rhetoric.

\section{Resumen}

En este artículo se discuten la historia, fundamentos y tensiones de la discriminación positiva, también conocida como acción afirmativa, en tanto concepto que se ha ido posicionando como un marco desde el que se han desarrollado acciones tendientes a disminuir las desigualdades sociales y aumentar las oportunidades en el ámbito educativo a distintos niveles de nuestras realidades globales como mundo contemporáneo. Las universidades, a nivel internacional, no se han quedado al margen, y han implementado acciones para mejorar el acceso, retención y egreso de estudiantes indígenas. Se propone aquí una reflexión crítica sobre estas acciones, como política y discurso, que permita ampliar nuestra mirada para reducir las brechas educativas teniendo como marco el concepto de discriminación positiva.

Palabras clave: universidades, trayectorias educativas, políticas educativas, estudiantes indígenas, retórica.

\section{Introducción}

En este artículo interesa abordar la discusión contemporánea en torno al concepto de discriminación positiva, pues ello permite visualizar su impacto y alcances para orientar el desarrollo de una política de educación superior para estudiantes indígenas en los países de América Latina. Con ello estoy suponiendo, desde el principio, que hay efectos beneficiosos derivados de las medidas de discriminación positiva. Sin embargo, es difícil, quizás imposible, determinar las relaciones causales directas entre las medidas de discriminación positiva y sus efectos sobre el desempeño de los estudiantes. Así, una extensa literatura internacional en educación superior indígena identifica factores vinculados a la experiencia educativa de 
estudiantes indígenas en educación superior que plantean barreras o afectan su éxito, a pesar de que ellos hayan ingresado a la educación superior como parte de acciones de discriminación positiva (por ejemplo, los estudios de Nakata et. al. sobre indígenas en Australia).

Sin embargo, la dificultad para poder analizar la relación causal entre la implementación de medidas de discriminación positiva y su impacto en la trayectoria educativa de estudiantes indígenas no implica que su efectividad no pueda ser analizada. A pesar de esta dificultad, aún permanecen abiertas a la investigación las preguntas legítimas sobre las medidas de discriminación positiva: ¿Ellas funcionan de la manera prevista? El reto es cómo explorar esa cuestión.

Mi proposición en este trabajo es que la eficacia de las medidas de discriminación positiva puede ser examinada a la luz de los términos de sus propias condiciones retóricas y administrativas. Examinar, aunque sea brevemente el discurso y prácticas de la acción afirmativa, aunque sea solo en la dimensión de las políticas nacionales a nivel de educación superior, abre caminos para realizar estudios que permitan ubicar discusiones epistemológicas en torno a la necesidad de cambiar conceptos y acciones ligadas a su ejercicio práctico. Así, podríamos preguntarnos, por ejemplo: (1) ¿una medida de discriminación positiva como la asistencia financiera a estudiantes, proporciona realmente condiciones razonables para que ellos cumplan con sus compromisos de estudio, a saber, suficiente tiempo para las tareas de estudio, condiciones de vida propicias para estudiar, capacidad para cumplir con los derechos educativos y financiar los recursos educativos necesarios?, y (2) ¿una política que apoye las disposiciones de ingreso especial también brinda suficiente apoyo para que los estudiantes superen la preparación académica insuficiente que los llevó a ingresar a través de estos programas de ingreso especial? Preguntas que parecen no estar vinculadas más que exclusivamente al ámbito educativo pero que, en función del examen aquí realizado, muestran la epistemología del discurso y prácticas de discriminación racial, étnica y social en todos los niveles de la interacción humana.

Formular este tipo de preguntas permite cuestionar, entonces, a las medidas de discriminación positiva y preguntarse si éstas posibilitan o limitan las oportunidades de acceso, participación, retención y resultados exitosos de los estudiantes indígenas. Pero no solo como un tema de políticas educativas, sino como un tema incluso de derechos humanos básicos. Ampliar nuestras perspectivas humanistas sobre los asuntos de distribución de recursos y oportunidades justas para todos se convierte, al final, en punto de una agenda de política pública del conocimiento social. La cuestión de la efectividad puede reorientarse, entonces, hacia las condiciones de incorporación y despliegue de medidas de discriminación positiva como "condicionantes" que promueven posibilidades o limitaciones para los estudiantes indígenas que ingresan a la educación superior.

\section{El concepto de discriminación positiva: historia, fundamentos y tensiones}

En su forma más simple, las medidas de discriminación positiva -también referidas como acción afirmativa o acción positiva en algunos lugares- tienen por objeto dar consideraciones especiales a los individuos sobre la base de su pertenencia a un grupo social que ha sido identificado sobre alguna base de desventaja en relación con otros grupos de la sociedad (Wang. Positive discrimination in education). Históricamente, el concepto de discriminación positiva surgió como respuesta a los esfuerzos por eliminar la discriminación en la segunda mitad del siglo XX tras el establecimiento de las Naciones Unidas y la Declaración Universal de Derechos Humanos. La Carta de los Derechos Humanos establece la igualdad de derechos y libertades de todo ser humano sin distinción alguna de raza, color, sexo, idioma, religión, opinión política o de otra índole, origen nacional o social, Estado. A todos los Estados Nacionales se les animó a observar estos 
derechos mediante medidas progresivas, partiendo de la premisa de la igualdad ante la ley para todos los ciudadanos.

Se atribuye a la India ser la primera nación que instituyó las bases para la discriminación positiva (Moses. Moral and instrumental rationales for affirmative action in five national contexts. Bacchi. Policy and discourse: challenging the construction of affirmative action as preferential treatment). Zachariah señala que ya en 1950 la Constitución Republicana de la India incluía artículos que prohibían la "discriminación por parte del Estado o los ciudadanos por motivos de religión, raza, casta, sexo o lugar de nacimiento" (Zachariah 1972:18). Según Zachariah, esta posición se construyó, hasta cierto punto, en los intentos anteriores del siglo XIX de abordar los niveles extremos de discriminación experimentados por grupos tales como los Intocables y otras castas y tribus inferiores, a través de la provisión de arreglos especiales para su protección y promoción social. También implicaba el reconocimiento temprano de que la discriminación ilegal en el sentido formal no superaría con facilidad las creencias inherentes y las prácticas de discriminación culturalmente integradas contra algunos grupos, a menos que se adoptaran medidas adicionales.

La justificación más comúnmente aceptada para las medidas de discriminación positiva es que la pertenecía a un grupo desfavorecido que privaba a sus miembros de la igualdad de oportunidades disponibles para otros miembros de la sociedad. Sin embargo, tal como señala Wang, en la práctica este argumento puede conducir a mecanismos de discriminación positiva basados en la "necesidad individual sin referencia a las características adscriptivas [derivadas de la pertenencia a un grupo, donde] el razonamiento grupal también se requiere" (Wang 1983:192). Según Wang, los derechos de los grupos a las medidas de discriminación positiva proceden de tres motivos principales: a) discriminación negativa histórica significativa (por ejemplo, afroamericanos, mujeres, pueblos indígenas), b) un estatuto especial basado en el reconocimiento de derechos inherentes o disposiciones constitucionales (por ejemplo, pueblos indígenas, castas y tribus de la India) y c) a veces para la integración política y económica de un grupo, a fin de estabilizar sociedades plurales (por ejemplo, Malasia).

La promoción y la defensa del concepto de discriminación positiva se ha sustentado, en parte, en discusiones sobre las barreras estructurales a la igualdad de oportunidades y los desafíos para superar los efectos históricos de exclusiones sostenidas de algunos grupos de personas de participación activa o igualitaria en el desarrollo cívico, y la vida política de la nación -una participación que ha dado oportunidades y beneficios a algunos grupos, mientras que instituye barreras a otros grupos. Sin embargo, en los Estados Unidos de América, por ejemplo, se reconoció pronto que, además de la discriminación estructural manifiesta, existían también formas más sutiles de discriminación que menoscababan la igualdad de oportunidades, a pesar del reconocimiento formal de la igualdad de todos los ciudadanos ante la ley. Moses atribuye la frase "acción afirmativa" (Moses 2010:13) al presidente Kennedy, quien en 1961 emitió la Orden Ejecutiva No. 10.925, exigiendo a todas las agencias gubernamentales que vayan más allá de la no discriminación pasiva para tomar medidas afirmativas para no discriminar en la contratación. Las leyes que prohíben la discriminación que se basan en la no discriminación pasiva no garantizan necesariamente resultados reales o no discriminatorios en la práctica.

La justificación de la discriminación positiva puso en tela de juicio la suposición democrática liberal de que la igualdad formal era suficiente para proporcionar igualdad de oportunidades a todos: "sin tener en cuenta la historia, el contexto y la discriminación pasada o presente" (Moses 2010:222). Ya en 1965, la defensa del presidente Johnson de la acción afirmativa desplegó más argumentos para subrayar esta razón; a saber, que la igualdad de oportunidades no surge necesariamente de la igualdad de trato de todos 
los individuos: "No tomas a una persona que ha sido trabada por las cadenas y lo liberas y luego dices 'eres libre de competir con los demás' y todavía cree [que usted] está siendo justo" (Bacchi 2004:132). Al extender el interrogatorio de la suposición subyacente de una igualdad formal que se basaba en la no discriminación pasiva, Young sugiere que "si la discriminación sirve a los propósitos de socavar la opresión de un grupo, puede no solo ser permitida, sino moralmente requerida" (Young, citado en Moses 2010:222).

Estas posiciones aparentemente contradictorias -estableciendo la discriminación positiva como un medio para lograr la no discriminación- se expresan a través de diferentes declaraciones dentro de las constituciones, la legislación, la política o las directivas de un número significativo de naciones en todo el mundo. No es de extrañar que esta paradoja sirva de base para los desafíos que se plantean constantemente a las medidas de discriminación positiva, porque perpetúa un trato discriminatorio, instituye formas de discriminación inversa que ponen en desventaja a los grupos sociales favorecidos y/o prevé el trato preferencial de algunos grupos otros y, como tal, socava la igualdad de oportunidades y la igualdad ante la ley. Un intento temprano en algunos lugares para resolver este paradójico estancamiento fue conducido "al ubicar los programas de acción afirmativa como exenciones a los estatutos contra la discriminación, indicando que debían ser considerados excepcionales, temporales y cuestionables en la ley" (Bacchi 2004:133). Como se puede observar, el cuestionamiento al concepto de discriminación positiva y sus prácticas asociadas ha sido -entonces- de suyo importante para ampliar las perspectivas de instrumentación política y argumentación retórica.

Además de los casos de India y Estados Unidos de América, ha sido en las democracias liberales donde se han instituido mecanismos de discriminación positiva: "existe una evidente tensión entre el principio de igualitarismo basado en la igualdad de oportunidades individual y las reivindicaciones de grupos de personas a favor tratamiento basado en su identidad histórica o posición frente a un territorio o país" (Wang 1983:193). Por lo tanto, la aceptación de un grupo por parte de la sociedad en general, como objetivo de medidas de discriminación positiva en el empleo y la educación, con mayor frecuencia también gira en torno a las desventajas socioeconómicas y educativas. Para los opositores de la discriminación positiva, esto plantea la pregunta de por qué tales medidas no pueden ser argumentadas sobre la base de la necesidad individual y así excluir la necesidad de socavar el principio de igualitarismo, un principio democrático fundador. Situado alrededor y entre estas posiciones polarizadas, es un debate complejo y discutido en curso en los niveles de la filosofía, la teoría, el derecho y la práctica.

Desde su surgimiento hasta el presente, la aceptación y defensa del concepto de discriminación positiva para grupos definidos de personas ha requerido, en primer lugar, el apoyo de diversos argumentos que pueden justificarlo junto con principios antidiscriminatorios; en segundo lugar, los motivos de elegibilidad para los grupos que afirman la necesidad de discriminación positiva (o tienen esa necesidad afirmada en su nombre); y en tercer lugar, un grado de aceptación pública de la misma como una redistribución justa de los recursos sociales que apoya, en lugar de socavar, los principios igualitarios.

\section{Discriminación positiva y educación superior}

Junto con el empleo, la educación superior es un sitio primario donde la discriminación positiva se ha instituido en diversas naciones a lo largo y ancho del globo. En la educación superior, las medidas de discriminación positiva tienen como objetivo dar especial consideración a los individuos por su pertenencia a un grupo que se ha identificado como sub-representado en la educación superior debido a factores asociados con la desventaja. Esta desventaja puede reconocerse sobre la base de algunos criterios 
especificados, como género, clase social / ingreso, racial, étnico, idioma, minoría, estado geográfico o estado histórico.

Para ser más que instrumentos retóricos, las medidas de discriminación positiva requieren la implementación de mecanismos concretos para lograr sus propósitos. A escala internacional, los argumentos de discriminación positiva parecen dirigirse principalmente hacia prácticas ajustadas de selección y admisión a la educación superior. Esto se entiende como una forma directa de proporcionar acceso, reconociendo las barreras estructurales a la igualdad de oportunidades educativas. En la educación superior, la prueba de la igualdad de oportunidades para todos se ha evaluado a menudo mediante la aspiración de representación proporcional de la población de grupos desfavorecidos designados en la población estudiantil. El establecimiento de cuotas numéricas o lugares reservados para grupos particulares (por ejemplo, India, Malasia) o el desvío de procesos competitivos normales y estándares de entrada calificados (por ejemplo, Australia) son dos enfoques comunes. Sin embargo, las medidas de discriminación positiva pueden expresarse a través de una serie de prácticas adicionales o alternativas, tales como programas de asistencia financiera designados para grupos determinados, provisiones de apoyo académico más allá de las disponibles para todos los estudiantes y desarrollo de políticas especiales o instituciones especiales para el grupo designado. En esencia, las medidas de discriminación positiva en la educación superior alteran la base tradicional de la selección individual basada en el mérito competitivo como condición para la participación en la educación superior (Wang. Positive discrimination in education).

A partir del examen de las justificaciones de la discriminación positiva desplegadas en la educación superior en cinco contextos nacionales, Moses sostiene que éstas se dividen en cuatro categorías de argumento: remedio de la discriminación en el pasado; argumentos económicos para que las personas desfavorecidas contribuyan a las economías nacionales; los argumentos de diversidad que destacan los beneficios educativos y sociales que derivan de la inclusión de la diversidad social en la educación superior; y la justicia social, que hacen hincapié en los beneficios sociales individuales y más amplios del acceso equitativo a la educación superior para su inclusión en las democracias participativas, que dependen de los esfuerzos concertados de una ciudadanía comprometida. Moses los clasificó como dos justificaciones fundamentales: instrumental y moral. Las razones instrumentales hacen hincapié en la discriminación positiva como un medio para llegar a un fin, "como proporcionar a la sociedad más trabajadores de grupos desfavorecidos o hacer que las instituciones de educación superior sean más diversas" (Moses 2010:218). Las justificaciones morales apelan a creencias más profundas sobre lo que es correcto y bueno y cómo se debe tratar a la gente; estos pueden ser hacia atrás o hacia adelante. Así, él citó las categorías "compensatorias" e "integradoras" de Anderson como ejemplos justificativos de justificaciones retrospectivas y progresistas: la primera es un "remedio hacia atrás" para la discriminación [pasada]; el segundo mira hacia adelante "para desmantelar las barreras actuales a la igualdad de oportunidades" (Moses 2010:218).

Se han resumido brevemente arriba las tensiones filosóficas generales que se involucran a través de los argumentos a favor y en contra de la discriminación positiva como principio. En la educación superior, los críticos de la discriminación positiva también han hecho argumentos basados en la percepción de los efectos educativos de las prácticas de discriminación positiva. Fischer y Massey, por ejemplo, han resumido los tres principales argumentos desplegados por los que se oponen a las prácticas de discriminación positiva en relación con las admisiones universitarias en los Estados Unidos de América: 
“(1) la acción afirmativa constituye una discriminación inversa que disminuye las probabilidades de admisión para los 'mejores' estudiantes blancos calificados;

(2) la acción afirmativa crea un desajuste entre las habilidades del estudiante y las habilidades requeridas para el éxito en las universidades selectivas, estableciendo así a los beneficiarios para el fracaso;

(3) la acción afirmativa estigmatiza a todos los miembros del grupo objetivo como no cualificado, lo que resulta en desmoralización y desempeño deficiente, independientemente de las calificaciones individuales" (Fischer y Massey 2007:532).

Las afirmaciones negativas sobre la discriminación positiva han producido intentos de investigar sus efectos, incluidos los efectos sobre posibles retiros de medidas de discriminación positiva (por ejemplo Wang 1983, Hsia. Limits of affirmative action, Fischer y Massey 2007, Francis y Tannuri-Pianto. Using Brazil's racial continuum to examine the short-term effects of affirmative action in higher education).

Es difícil de extraer conclusiones generales de los estudios discretos, pues diferentes contextos nacionales, sociales y políticos condicionan los supuestos y la estructuración de éstos. Sin embargo, en un estudio estadounidense de Bowen y Bok, la eliminación de la acción afirmativa fue calculada para "elevar la tasa de admisión promedio de los estudiantes blancos en 2 puntos porcentuales, de un $25 \%$ a un $27 \%$, un pequeño margen" (Bowen y Bok 1998:222). Sobre la base de estos hallazgos, Loury proporcionó una perspectiva a los que apoyan la posición "contra": “¿Por qué, entonces, toda la energía... angustia... palpitante... clamor, por qué toda la preocupación de que América está siendo encallada, que nuestros estándares están siendo destrozados, que los bárbaros están en la puerta? ¿Por qué esa resistencia cuando, como indican los datos del libro de Bowen y Bok, el límite de la jerarquía racial está siendo borrado solo un poco por los escasos pocos estudiantes negros que, al margen y debido a las prácticas de acción afirmativa de las universidades, se están introduciendo en los cuadros de liderazgo de los Estados Unidos de América?" (Loury, citado en Moses 2010:222).

Como ha señalado Wang, "la discriminación positiva hace que la selección educativa sea un asunto abiertamente político" (Wang 1983:199). Sin embargo, algunos han ofrecido críticas a la discriminación positiva en el acceso a la educación superior porque la discriminación positiva puede producir proporciones relativamente pequeñas de élites educadas dentro de grupos sociales desfavorecidos grupos que siguen siendo abrumadoramente pobres o desfavorecidos debido al descuido de mejorar la educación en los niveles de la escuela primaria y secundaria. Una inferencia es que la discriminación positiva en la educación superior, al descuidar la atención a los resultados equitativos en la escolarización, es posiblemente un medio ineficiente de redistribuir los recursos para la igualdad de oportunidades educativas de todos. Sin embargo, a raíz de esta preocupación, la falta de atención a los resultados escolares de los grupos a los que se dirigen las medidas de discriminación positiva en el nivel de educación superior parece estar implicada en la necesidad continua de estas medidas para mejorar el acceso a la educación superior.

Si bien la necesidad continua que se ha utilizado para argumentar que la discriminación positiva en la educación superior es un fracaso, también pone en duda la suficiencia del principio de igualdad de oportunidades, cuando el resultado de su aplicación en la educación sigue siendo debatida. Sin embargo, Moses concluye: "Como la acción afirmativa en la educación superior sigue siendo debatida en todo el mundo, una cosa es clara. El mayor acceso y la oportunidad que viene con la política de acción afirmativa benefician a los estudiantes que por una razón u otra están en desventaja en la sociedad. Esto no es una cosa pequeña" (Moses 2010:224). 
Por tanto, el debate en torno a la "efectividad" de la discriminación positiva requiere una mirada que supere la relación binaria entre "éxito" y "fracaso", puesto que los resultados académicos y las trayectorias educativas de estudiantes indígenas forman parte de un entramado más complejo, que no se define ni culmina con la implementación de políticas de discriminación positiva.

\section{La importancia de las particularidades históricas en los contextos nacionales para los fundamentos y prácticas de la discriminación positiva}

Si bien Moses aboga firmemente por una movilización más amplia de la lógica de la justicia social en la educación superior, su análisis también apoya la idea de que las particularidades de los contextos nacionales determinarán en qué medida predominan los fundamentos y que pueden ser útiles para la justificación de las acciones de discriminación positiva. Así, examinó cinco contextos nacionales diversos Francia, Brasil, India, Sudáfrica y los Estados Unidos de América- para poner de relieve cómo los argumentos que justifican las acciones de discriminación positiva en relación con la educación superior son moldeados por la historia y las particularidades de los contextos sociales, las identidades y los valores de cada nación. El análisis de Moses es útil para los propósitos de este artículo porque, como dice: "Cada uno de estos países puede aprender unos de otros. En última instancia, cómo se resuelven las disputas de acción afirmativa afectará la vida de muchos estudiantes insuficientemente representados y de estas sociedades democráticas" (Moses 2010:221). Por ejemplo, nuestro autor tomó en cuenta las autopercepciones o identidades nacionales que dan forma a los discursos y debates de acción afirmativa en estos países respectivos: "Para Francia, es una ideología centrada en la unidad, el universalismo y la asimilación de los inmigrantes en la cultura francesa... La India está pasando de la ideología casta de la desigualdad inherente a una conciencia social de desventaja sistémica y los beneficios de la diversidad en la vida social... Sudáfrica se ha movido gradualmente de la ideología apartheid de la desigualdad a la reconciliación y la unidad nacional... La ideología estadounidense se centra en la nación como una democracia liberal... y el ideal nacional de Brasil ha sido el de una democracia racial caracterizada por la creencia de que la raza no importa social o políticamente" (Moses 2010:213).

Estas percepciones de la identidad nacional subrayan las percepciones correspondientes de las desigualdades en las oportunidades educativas y el acceso a la educación superior y producen diferentes razones para las medidas de discriminación positiva. Por ejemplo, Moses sostiene que en Francia las actitudes y políticas oficiales apoyan la asimilación de los inmigrantes (que constituyen la creciente diversidad racial, cultural y religiosa de la nación) y su posicionamiento como francés. Así, en Francia, no se ha movilizado una creciente diversidad cultural para ampliar lo que significa ser francés, como intentó hacer la política multicultural en Canadá y Australia, por ejemplo. Como resultado, en Francia, los resultados socioeconómicos y educativos desiguales de los grupos de inmigrantes han sido lentos en afirmarse como vinculados a prácticas institucionales o discriminatorias. De acuerdo con él, aunque ha habido una ley contra la discriminación desde 1972, no ha habido directivas de acción afirmativa a nivel gubernamental, a pesar de que las medidas de acción afirmativa han surgido dentro de algunas instituciones de educación superior. Esto demostraría cómo la clase y la falta de recursos financieros siguen siendo las explicaciones duraderas de la inequidad educativa. Las cuestiones de discriminación por motivos de raza, color, cultura, religión o idioma siguen sumergidas debido a la creencia de que no deben importar. El color y la raza 'ciega' siguen siendo una virtud francesa nacional y una demostración de su ethos igualitario.

Sudáfrica, por otra parte, desde la caída del régimen del apartheid en 1992, cuya ideología se adhirió a la noción de desigualdad racial inherente, ha hecho de la raza (y el género) la base primaria de la política de 
acción afirmativa. La historia de Sudáfrica requiere una redistribución a gran escala del capital y de los recursos sociales a favor de la mayoría. Con el $79 \%$ de la población clasificada como 'negros', solo el $1 \%$ pasa a la educación superior. La acción afirmativa se fundamenta en la Constitución y en una Ley de Educación Superior de 2006 que ordena a las universidades aumentar las admisiones de "negros" y otras categorías sub-representadas. A pesar de esto, Moses reporta preocupaciones constantes en las discusiones y debates nacionales sobre los conflictos entre las razones de la discriminación positiva y el discurso de la reconciliación. La necesidad de una redistribución tan grande de los recursos sociales pone de relieve los privilegios anteriores de los blancos y la pérdida de estos privilegios puede ser presentada de nuevo en términos de una sanción contra los blancos, en particular los blancos socioeconómicos más bajos.

En contraste con estos dos países, según Moses, la narrativa nacional de Brasil como una "democracia racial" ha sido expuesta como enmascaramiento de la causa de "niveles dispares de logro educativo por raza, etnia y clase social" (Moses 2010:217). Citando a Htun, quien caracterizó la autopercepción de Brasil "no como un pueblo compuesto de distintas" razas "sino como una raza nacional multicolor", Moses revela cómo las discusiones sobre las medidas de discriminación positiva han girado en los debates sobre la ponderación relativa dada la importancia que la raza juega en la inequidad educativa, en oposición a la clase. La raza es considerada una cuestión más divisiva que la clase por los opositores de la discriminación positiva, mientras que los partidarios sostienen que la raza es una cuestión social importante que debe ser reconocida. Como en otros lugares, cuando el color o la ceguera racial apoyan las percepciones y los principios del igualitarismo, las desigualdades en oportunidades educativas y acceso se ven a través del prisma menos conflictivo de desventaja económica, incluso cuando se entiende la relación entre raza y desventaja económica.

Los debates que se desarrollan de acuerdo con las particularidades e historias nacionales que producen la consideración de medidas de discriminación positiva son un recordatorio de "cómo la acción afirmativa flexible, adaptable y contextual ha estado de hecho en diferentes partes de la palabra. Su ambigüedad y adaptabilidad son tanto su fuerza como su fragilidad. No es una fórmula fija para la acción gubernamental transportable de un país a otro, ni es un arreglo legal o constitucional preciso de aplicación universal. Sin embargo, tiene una característica principal. Dondequiera que funcione y cualquiera que sea su terminología, implica una intervención gubernamental deliberada y focalizada que tenga en cuenta la realidad de la raza para tratar y superar los problemas asociados con la raza" (Sachs, citado en Moses 2010:224).

\section{Discriminación positiva como política y discurso}

La discriminación positiva, como se ha expuesto anteriormente, se presenta como una proposición conceptual para lograr una intención progresivamente intencionada a través de un conjunto deliberado de razones y principios de acción. También puede entenderse como política; por ejemplo, la política que se crea y la acción mediante una voluntad política para servir a intereses y fines particulares. Bacchi ha posicionado la política como discurso y la acción afirmativa como "parte de una competencia discursiva, no como algo fundamentado en principios" (Bacchi 2004:131). A través de este posicionamiento, los argumentos que exponen las tensiones en los debates a favor y en contra de la discriminación positiva pueden ser examinados más de cerca.

Bacchi aboga por el examen de las premisas de los conceptos subyacentes a los argumentos. Por ejemplo, revela cómo la noción dominante de discriminación positiva como tratamiento preferencial representa a 
los sujetos de la política de discriminación positiva como el problema: los 'objetivos' o los sujetos de la política de discriminación positiva son los que deben cambiar. Además, sostiene, "la implicación es que ellos no podrían haber alcanzado este nivel de éxito por sí mismos" (Bacchi 2004:135), construyendo así grupos objetivo en términos de déficit. Además, la representación de los grupos objetivo como el "problema" sitúa las soluciones de trato especial como actos de generosidad: "Aquellos que están dispuestos a hacer provisiones 'especiales' para ayudarles a superar el obstáculo de su 'atraso' aparecen como benevolentes y ciertamente como benéficos. Las condiciones por las que estos 'benefactores' llegaron al poder y mantienen el poder permanecen sin ser problematizadas. La acción afirmativa se convierte en una especie de caridad y la pregunta es: ¿cuánta 'ayuda' necesita la 'desfavorecida'? ¿Y cuánta 'ayuda' es 'permisible'?"' (Bacchi 2004:132).

A juicio de Bacchi, al constituir los sujetos de la discriminación positiva como problema, se permite que el statu quo permanezca incontestable en cualquier sentido estructural real, incluso cuando los partidarios de la conceptualización de la discriminación positiva como trato preferencial "se comprometen con una profunda transformación estructural" (Bacchi 2004:129). Sin embargo, como ella continúa revelando, el problema se puede considerar de otras maneras. En lugar de desafiar las disposiciones de acceso "especiales" como un beneficio especial que denota un trato preferencial, la noción de acceso basado en el mérito como un proceso "justo" puede ser cuestionada. Bacchi llama la atención sobre otros teóricos que desafiaron suposiciones y presentaron otras representaciones del problema, en lo que se refiere a los procesos de nombramientos de empleo. Los estándares y procesos de la base meritocrática de los nombramientos fueron postulados como no justos u objetivos, pero sometidos a los prejuicios culturales y procesales de quienes los instituyeron y por medio de los cuales mantuvieron sus bases de autoridad y poder. Estas cargas podrían aplicarse de manera similar a los estándares y procedimientos de educación superior para la selección para admisión y para las prioridades en el desarrollo de programas e investigaciones. Además, Bacchi expone los desafíos a la base condicional de las medidas de acción afirmativa como una exención a la ley contra la discriminación. Esta lógica, afirma, "asume que las reglas sociales son generalmente justas, pero que algunas personas se enfrentan a actitudes perjudiciales y / o bloqueos incidentales que los impiden" (Bacchi 2004:133). Ella se basa en el trabajo de otros para apoyar su argumento: "Black ofrece un análisis muy diferente del problema. Él desafía la noción que la ley es sobre todo un asunto de las reglas y que la discriminación es una aberración. Demuestra que muchos factores influyen en quién ganará y en cuál será el castigo. Si la discriminación no es una aberración, en su opinión, tratarla como tal oculta necesariamente la forma en que las «diferencias sociales impregnan la ley». De ello se desprende que los intentos de remediar este privilegio no son discriminaciones, positivas o de otro tipo, sino esfuerzos por hacer justicia" (Bacchi 2004:133).

Para Bacchi, sin embargo, el problema "no es la condición de ser objetivo, sino la construcción discursiva de la acción afirmativa como trato preferencial" (Bacchi 2004:135). Como señala, en referencia a la caracterización de los aborígenes como desfavorecidos por un lado y, por otro lado, como beneficiarios privilegiados de la acción afirmativa, "otros grupos que son apuntados para asignaciones regulares de fondos gubernamentales, como los veteranos de guerra, los agricultores de trigo y los fabricantes de automóviles, no se caracterizan de esta manera" (Bacchi 2004:135). Así, algunas formas de trato preferencial son aceptables y otras se consideran excesivas si interfieren con procedimientos considerados ya bastante equitativos. De este modo, la conceptualización de la acción afirmativa como "trato preferencial" limita los tipos de programas considerados aceptables.

Las detalladas indagaciones genealógicas de Bacchi le llevan a concluir que la acción afirmativa "forma parte de un marco de significado que deja básicamente sus supuestos de igualdad de oportunidades en su 
sitio" (Bacchi 2004:134). Hablando de empleo en lugar de acceso a la educación superior, afirma "la conceptualización de la acción afirmativa como 'trato preferencial' configura, por lo tanto, las discusiones de una manera que trata de deslegitimar la reforma y de hacerla ineficaz. Dentro de este marco se hace imposible cuestionar las normas aplicadas a aquellos que actualmente tienen cargos de poder y autoridad, o que siguen siendo utilizados en la contratación y promoción. Es imposible llamar la atención sobre las reglas de fondo que protegen el statu quo social. De hecho, el concepto parece encajar con esas reglas. El argumento de que los miembros de grupos desfavorecidos necesitan 'ventajas' acepta implícitamente que en general la igualdad de oportunidades funciona de manera justa y equitativa” (Bacchi 2004:136).

Para Bacchi el dilema es claro: "mientras las reglas de fondo de una sociedad no sean cuestionadas, la 'solicitud' de recursos para los miembros de los grupos sigue siendo una especie de caridad otorgada por personas benéficas a individuos representativos de algunos grupos" (Bacchi 2004:139).

En el nivel superficial de la descripción y el análisis, las suposiciones que sustentan la manera en que las discusiones sobre la discriminación positiva son moldeadas y conducidas, no se revelan necesariamente en su propio cuestionamiento. Esto, a pesar de que en la actualidad la discriminación positiva se ha posicionado como un concepto internacional respaldado por los derechos humanos, que permite la puesta en práctica de acciones discriminatorias beneficiosas como un medio para superar los efectos de la discriminación sistémica negativa pasada contra ciertos grupos sociales. Por este motivo, se requieren investigaciones más profundas para desafiar los significados comúnmente aceptados y expresados en los debates y discusiones sobre la discriminación positiva, como por ejemplo principalmente aquellos que se refieren a ésta como un instrumento "efectivo" de cambio, así como también las expectativas que se generan en torno a las acciones implementadas "en su nombre". Una mirada crítica y reflexiva sobre la discriminación positiva, permitirá superar la permanente tensión entre lo que se espera "idealmente" como resultado y lo que, efectivamente, pueda lograr como marco de acción tendiente a superar los efectos negativos y las brechas de los grupos sociales menos favorecidos.

\section{Conclusión}

Demostrar la importancia del debate conceptual en torno a la discriminación positiva en términos de su materialidad en políticas públicas es crucial para modificar prácticas institucionales de diversas índoles: a nivel educativo, a nivel gubernamental y a nivel comunitario. Tanto las poblaciones indígenas como las no indígenas necesitan visualizar, en todas sus dimensiones, los efectos variados de las medidas de discriminación positiva. Así, es prioritario mirar nuestra realidad latinoamericana desde una perspectiva ampliada de derechos humanos que ubique analíticamente la urgencia de criticar los legados conceptuales y las coordenadas geográficas mediante las cuales se orientan las políticas nacionales de educación superior y los proyectos posibles de discriminación positiva.

Comparar naciones y estar conscientes de las diferentes tradiciones que forman una retórica que legitima prácticas de desigualdad social a niveles internacionales, nos ha de permitir generar condiciones óptimas para instrumentar acciones efectivas para los objetivos democráticos de superar el rezago y posicionar políticas cuyos fundamentos epistemológicos se sostengan por el análisis histórico crítico de los conceptos que las sustentan. Deseo que el breve ejercicio práctico comunicado en este trabajo ofrezca algunas luces guía para seguir caminos innovadores en esa dirección.

Las poblaciones de nuestros pueblos originarios no han accedido mayoritariamente a los beneficios de la educación superior, en comparación con otros grupos sociales, a pesar de los discursos de equidad e 
igualdad de los tecnócratas planificadores de las políticas sociales y educativas. Epistemológicamente hablando eso significa que no ha habido repercusiones conceptuales de los debates sobre la democracia equitativa en términos de justicia. Está aún lejana la etapa social en la que las poblaciones indígenas participen de la ciencia y la tecnología occidental, incluso a nivel del respeto a sus conocimientos ancestrales, en la perspectiva de un concurso democrático.

Identificar los caminos posibles de soluciones viables para superar discursos institucionalizados que, por sobre las bases de un discurso crítico, mantienen situaciones de desigualdad, es una tarea ingente para todos aquellos investigadores que estamos preocupados por la discriminación en cualquiera de sus formas. ¿Qué hacer frente al mundo cambiante que nos exige no aceptar ideologías de recambio que solo representan transformaciones a nivel superficial sin que las estructuras que definen al acontecer mundano de hoy se transformen? Es tarea de una investigación como la aquí realizada desenmascarar los patrones discursivos que sostienen el patrón de injusticia social actual.

No es suficiente cuestionar las prácticas de acciones afirmativas en las instituciones de enseñanza superior en términos de su ineficacia para reducir las brechas educativas, pues debemos ir a los fundamentos epistemológicos e incluso ontológicos que posibilitan su existencia como discurso público y generador de políticas. Es definitivo reconocer, descifrar, analizar y deconstruir la epistemología del discurso y prácticas de discriminación racial, étnica y social en todos los niveles de la interacción humana a fin de proponer nuevas modalidades de acción que fructifiquen en armonías con justicia social.

El análisis conceptual es una tarea ingente que la epistemología de nuestro siglo XXI no puede eludir. En otras palabras: la crítica radical a la conceptualización de la discriminación positiva como trato preferencial, fundada no solo en sus discursos, sino también en las prácticas culturales, legales e institucionales de la sociedad concreta que se analiza, debe ser un compromiso de la investigación social responsable y comprometida con la transformación.

\section{Agradecimientos}

Este trabajo forma parte del proyecto UTA Mayor 2017 (3763-17) "Narraciones biográficas y factores vinculados al desempeño académico en las trayectorias educativas de estudiantes Indígenas de la región de Arica y Parinacota".

\section{Bibliografía}

Bacchi, C. 2004. Policy and discourse: challenging the construction of affirmative action as preferential treatment. Journal of European Public Policy 11(1): 128-146 https://doi.org/10.1080/1350176042000164334

Bowen, W.G. y Bok, D. 1998. The shape of the river: long-term consequences of considering race in college and university admissions. Princeton: Princeton University Press.

Fischer, M.J. y Massey, D.S. 2007. The effects of affirmative action in higher education. Social Science Research 36(2): 531-549 https://doi.org/10.1016/i.ssresearch.2006.04.004

Moses, M.S. 2010. Moral and instrumental rationales for affirmative action in five national contexts. Educational Researcher 39(3): 211-228 https://doi.org/10.3102/0013189X10365086 
Wang, B. 1983. Positive discrimination in education: a comparative investigation of its bases, forms, and outcomes. Comparative Education Review 27(2): 191-203 https://www.jstor.org/stable/1187908

Zachariah, M. 1972. Positive discrimination in education for India's scheduled castes: a review of the problems, 1950-1970. Comparative Education Review 16(1): 16-29

https://www.jstor.org/stable/1186339

Recibido el 4 Jul 2018

Aceptado el 28 Oct 2018 